\title{
Depression Promotes the Onset of Irritable Bowel Syndrome through Unique Dysbiosis in Rats
}

Takeshi Takajo $^{1}$, Kengo Tomita ${ }^{1}$, Hanae Tsuchihashi ${ }^{2}$, Shingo Enomoto ${ }^{3}$, Masaaki Tanichi ${ }^{3}$, Hiroyuki Toda ${ }^{3}$, Yoshikiyo Okada $^{1}$, Hirotaka Furuhashi ${ }^{1}$, Nao Sugihara ${ }^{1}$, Akinori Wada ${ }^{1}$, Kazuki Horiuchi ${ }^{1}$, Kenichi Inaba ${ }^{1}$, Yoshinori Hanawa ${ }^{1}$, Naoki Shibuya ${ }^{1}$, Kazuhiko Shirakabe ${ }^{1}$, Masaaki Higashiyama ${ }^{1}$, Chie Kurihara ${ }^{1}$, Chikako Watanabe $^{1}$, Shunsuke Komoto $^{1}$, Shigeaki Nagao ${ }^{1}$, Katsunori Kimura ${ }^{2}$, Soichiro Miura ${ }^{1,4}$, Kunio Shimizu ${ }^{5}$, and Ryota Hokari ${ }^{1}$

${ }^{1}$ Division of Gastroenterology and Hepatology, Department of Internal Medicine, National Defense Medical College, Tokorozawa, ${ }^{2}$ Microbiome Group, Basic Microbiology Research Department, Food Microbiology Research Laboratories, R\&D Division, Meiji Co., Ltd., Hachiouji, ${ }^{3}$ Department of Psychiatry, National Defense Medical College, Tokorozawa, ${ }^{4}$ International University of Health and Welfare Graduate School, Tokyo, and ${ }^{5}$ Division of Behavioral Sciences, National Defense Medical College Research Institute, Tokorozawa, Japan

Background/Aims: Although studies using conventional animal models have shown that specific stressors cause irritable bowel syndrome (IBS), it is unclear whether depression itself causes IBS. Our aim was to establish a rat model to determine if depression itself promotes the onset of IBS and to elucidate the role of gut microbiota in brain-gut axis pathogenesis during coincident depression and IBS. Methods: Rat models of depression were induced using our shuttle box method of learned helplessness. Visceral hypersensitivity was evaluated by colorectal distension (CRD) to diagnose IBS. Gut microbiota compositions were analyzed using highthroughput sequencing. In the subanalysis of rats without depression-like symptoms, rats with posttraumatic stress disorder (PTSD) were also examined. Results: The threshold value of CRD in depressed rats was significantly lower than that in control rats. Microbial community analysis of cecal microbiota showed that the relative abundance of Clostridiales incertae sedis, the most prevalent microbe, was significantly lower in depressed rats than in control rats. The distribution pattern of the microbiota clearly differed between depressed rats and control rats. Neither visceral hypersensitivity nor the composition of gut microbiota was altered in rats with PTSDlike phenotypes. Conclusions: Our rat model of depression is useful for clarifying the effect of depression on IBS and suggests that depression itself, rather than specific stressors, promotes the onset of IBS. Further, we provided evidence that various psychiatric diseases, viz., depression and PTSD, are associated with unique gut microbiota profiles, which could differentially affect the onset and progression of coincident IBS. (Gut Liver 2019;13:325-332)

Key Words: Irritable bowel syndrome; Depression; Stress disorders, post-traumatic; Gastrointestinal microbiome

\section{INTRODUCTION}

Psychological stress is a major factor in health and well-being that causes many psychiatric disorders, such as depression and anxiety, which are associated with the development of some gastrointestinal diseases, including irritable bowel syndrome (IBS). It has been reported that individuals with a history of depression are at high risk of developing IBS, and indeed, a relatively large number of IBS patients have coincident depression. ${ }^{1,2}$ Therefore, it has been suggested that psychological stress might affect the brain-gut axis, ${ }^{2}$ resulting in motility disorders and hypersensitivity of the gastrointestinal tract. Although studies using conventional animal models have shown that specific stressors cause IBS to develop, it is not clear whether depression per se contributes to the development of IBS

Recent reports have also shown that gut flora play a role in pathogenesis in the brain-gut axis. ${ }^{3}$ Recent high-throughput microbial community analyses of gut flora have shown that there are significant differences in gut flora between normal subjects and patients with IBS. ${ }^{4}$ Therefore, some therapeutic attempts have been made to treat patients with IBS using probiotics. ${ }^{5}$ Furthermore, depressed patients also have different gut flora than normal subjects. ${ }^{6}$ These results suggest that there could be

Correspondence to: Kengo Tomita (https://orcid.org/0000-0003-2625-6445) ${ }^{\mathrm{a}}$ and Ryota Hokari (https://orcid.org/0000-0002-8511-2540)

Division of Gastroenterology and Hepatology, Department of Internal Medicine, National Defense Medical College, 3-2 Namiki, Tokorozawa 3598513, Japan

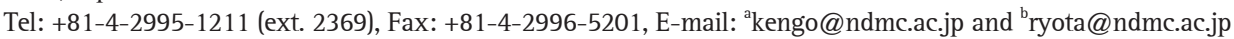

Received on June 28, 2018. Revised on August 26, 2018. Accepted on September 5, 2018. Published online February 12, 2019 pISSN 1976-2283 eISSN 2005-1212 https://doi.org/10.5009/gnl18296

(c) This is an Open Access article distributed under the terms of the Creative Commons Attribution Non-Commercial License (http://creativecommons.org/licenses/by-nc/4.0) which permits unrestricted non-commercial use, distribution, and reproduction in any medium, provided the original work is properly cited. 
some pathological correlations among gut flora, IBS, and depression.

In the present study, we first aimed to establish a rat depression model to clarify whether depression itself promotes the onset of IBS, and, if so, to determine the pathological mechanism. Second, we aimed to elucidate the role of gut flora in brain-gut axis pathogenesis in coincident depression and IBS using highthroughput microbial community analysis of gut flora in a rat model of depression.

\section{MATERIALS AND METHODS}

\section{Animals}

Seven-week-old male Wistar rats (CLEA, Tokyo, Japan) were used. The care and use of the laboratory animals were in accordance with the guidelines of the "Methods and welfare considerations in behavioral research with animals: report of a National Institutes of Health Workshop" published by the US National Institutes of Health (Bethesda, MD, USA) and with the guidelines of the animal facility at the National Defense Medical College (NDMC) in Japan. All experimental protocols were approved by the Animal Research Committee of the NDMC (No. 15089). Rats were housed in plastic cages at a density of 3 per cage and were maintained under a 12-hour light and dark cycle at a temperature of $24^{\circ} \mathrm{C}$ and with $55 \%$ humidity. The rats were fed standard laboratory chow (CLEA Japan, Tokyo, Japan), and food and water were available ad libitum.

\section{Establishment of a rat model of depression using the shuttle box method}

We used a shuttle box behavior analysis system as previously described. ${ }^{71}$ Seven-week-old male Wistar rats were used after acclimation for 7 days. The rats were given 60 inescapable foot shocks (current, $0.8 \mathrm{~mA}$; duration, 15 seconds; intertrial interval (ITI), $15 \pm 7.5$ seconds) without any conditioned stimulus (CS) in a dark shuttle box consisting of two compartments (Med Associates, Inc., St. Albans, VT, USA). Two weeks later, the locomotor activity of the rats was measured during a 5-minute adaptation period to the surrounding environment.

Then, an avoidance/escape task (AET) procedure was repeated 80 times (standby time, $15 \pm 7.5$ seconds) in the same shuttle box. In the AET procedure, after 5 seconds of light stimulation as a $\mathrm{CS}$, rats were electrically shocked (current, $0.8 \mathrm{~mA}$; maximum duration, 15 seconds) until they passed through the central gate into the next compartment of the box. As previously described, the rats were then assessed for learned helplessness (LH), ${ }^{12,13}$ which is thought to be a depression-like behavior corresponding to a rat model of depression. When the rats continued to allow exposure to electric shock by not passing through the central gate of the box in more than 10 out of 80 exposures, they were diagnosed with LH. Among the rats not diagnosed with LH (non-LH), some rats had both low activity (passing through the central gate less than 10 times) during the adaptation period and high activity (passing through the central gate more than 40 times) during the ITI and light stimulation (CS) periods. As this behavior could be regarded as a bidirectional behavioral pattern of activity and reactivity characteristic of posttraumatic stress disorder (PTSD), these rats were diagnosed with PTSD as previously described. ${ }^{7-9,11}$ Control group rats were not subjected to foot shocks or the AET procedure.

\section{Colorectal distension}

Visceral hypersensitivity was evaluated using colorectal distension (CRD), as previously described. ${ }^{14}$ In brief, a 4-cm long collapsible balloon was inserted $6 \mathrm{~cm}$ beyond the anus in each rat under anesthesia. Then, each rat was placed in a small Lucite cubicle (KN-325-A; Natsume Seisakusho Co., Ltd., Tokyo, Japan) and acclimated in an awake state for 30 minutes. The intraluminal pressure at which the abdominal muscles of the rat started contracting after the balloon was gradually pressurized was taken as the threshold CRD value. The procedure was repeated five times, and the threshold CRD value was measured by two observers blinded to the experimental conditions.

\section{High-throughput sequencing of the gut microbiota}

Fresh stool was taken from the cecum of each rat after CRD evaluation and stored at $-80^{\circ} \mathrm{C}$. Bacterial DNA was extracted from the stool samples using a QIAamp Stool Mini Kit (Qiagen, Valencia, CA, USA) as previously described. ${ }^{15}$ The V4 region of $16 \mathrm{~S}$ rRNA was amplified using region-specific primers (forward: 515F, reverse: 806rcbc33 52) and TaKaRa Ex Taq ${ }^{\circledR}$ (Takara Bio Inc., Osaka, Japan) as previously described. ${ }^{16}$ The polymerase chain reaction (PCR) amplicon was purified using a QIAquick PCR Purification Kit (Qiagen) according to the manufacturer's instructions. Library quantification, normalization, and pooling were performed according to the Sequencing Library qPCR Quantification Guide (Illumina, San Diego, CA, USA). The size and quality of the pooled libraries were ascertained using MultiNA (Shimadzu Corp., Kyoto, Japan). Following NaOH denaturation, the libraries were loaded into the MiSeq cartridge and sequenced on a MiSeqIII instrument (Illumina) according to the manufacturer's instructions. The sequence data were analyzed using the Quantitative Insights Into Microbial Ecology (QIIME) tool. ${ }^{17}$

\section{Statistical analyses}

All data are displayed as the mean \pm standard error of the mean (SEM). The CRD thresholds were analyzed using one-way analysis of variance followed by the Tukey-Kramer post hoc test. The relative abundances of cecal microbe groups were analyzed using the Kruskal-Wallis test followed by the Steel-Dwass post hoc test. All analyses were performed using JMP Pro, version 12.2.0 (SAS Institute Inc., Cary, NC, USA). Probability values less than 0.05 were considered statistically significant. 
Additionally, microbial diversity was analyzed with QIIME.

\section{RESULTS}

\section{Visceral hypersensitivity is induced in rats with LH}

The mean threshold CRD value for each rat was measured after the behavior test. The mean values were $29.07 \pm 1.07 \mathrm{~mm} \mathrm{Hg}$ in the control group, $21.42 \pm 1.57 \mathrm{~mm} \mathrm{Hg}$ in the LH group, and $28.43 \pm 1.49 \mathrm{~mm} \mathrm{Hg}$ in non-LH group. The value in the LH group was significantly lower than that in the control group, whereas there were no significant differences between the non-LH and control groups (Fig. 1).

\section{The composition of the gut microbiota is altered in rats with LH}

High-throughput microbial community analysis of the cecal microbiota at the phylum level for each rat showed that the relative abundance of Actinobacteria was significantly higher in the LH group than in the control group (Fig. 2). We next performed high-throughput microbial community analysis of the cecal microbiota at the family level for each rat and identified microbe families with relative abundances of $0.1 \%$ or greater (Fig. 3). The relative abundances of Lactobacillaceae, Turicibacteraceae, Peptostreptococcaceae, and Bifidobacteriaceae were significantly higher in the LH group than in the control group, whereas that of Clostridiales incertae sedis was significantly lower in the LH group than in the control group (Fig. 3). In addition, the relative abundance of S24-7 was significantly higher in the non-LH group than in the control group, while that of
Mogibacteriaceae was significantly lower in the non-LH group than in the control group (Fig. 3). Furthermore, we identified the rat cecal microbes with relative abundances of $0.1 \%$ or greater at the genus level (Table 1). The relative abundances of Lactobacillus, Turicibacter, Peptostreptococcaceae incertae sedis, and Bifidobacterium were significantly higher in the LH group than in the control group, whereas that of Clostridiales incertae sedis was significantly lower in the LH group than in the con-

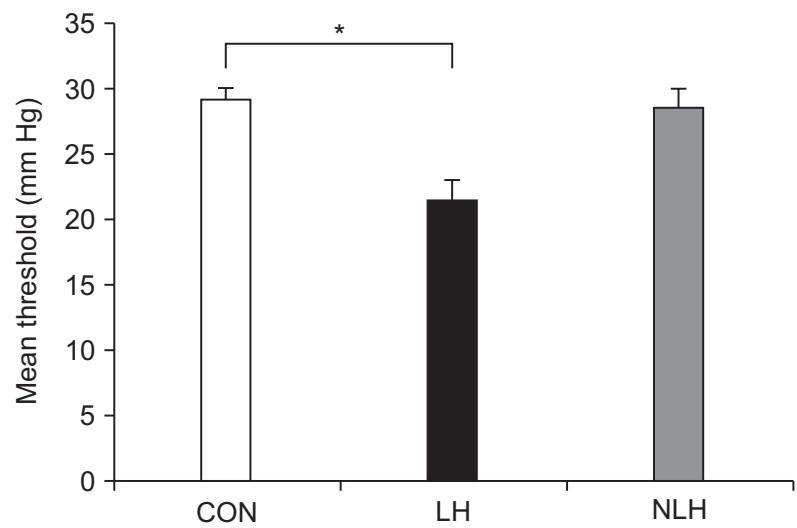

Fig. 1. Visceral hypersensitivity is induced in rats with learned helplessness (LH). The mean threshold value of colorectal distension in each rat (CON: 5 control rats subjected to neither foot shock nor the avoidance/escape task procedure; LH: 5 rats diagnosed with LH; NLH: 10 rats diagnosed as not having $\mathrm{LH}$ ) was measured after the behavioral test. The mean threshold value was $29.07 \pm 1.07 \mathrm{~mm} \mathrm{Hg}$ in the control group, $21.42 \pm 1.57 \mathrm{~mm} \mathrm{Hg}$ in the LH group, and $28.43 \pm 1.49$ $\mathrm{mm} \mathrm{Hg}$ in the NLH group. ${ }^{*} \mathrm{p}<0.05$ vs control rats. $\mathrm{p}$-values obtained via one-way analysis of variance with Tukey post hoc test.
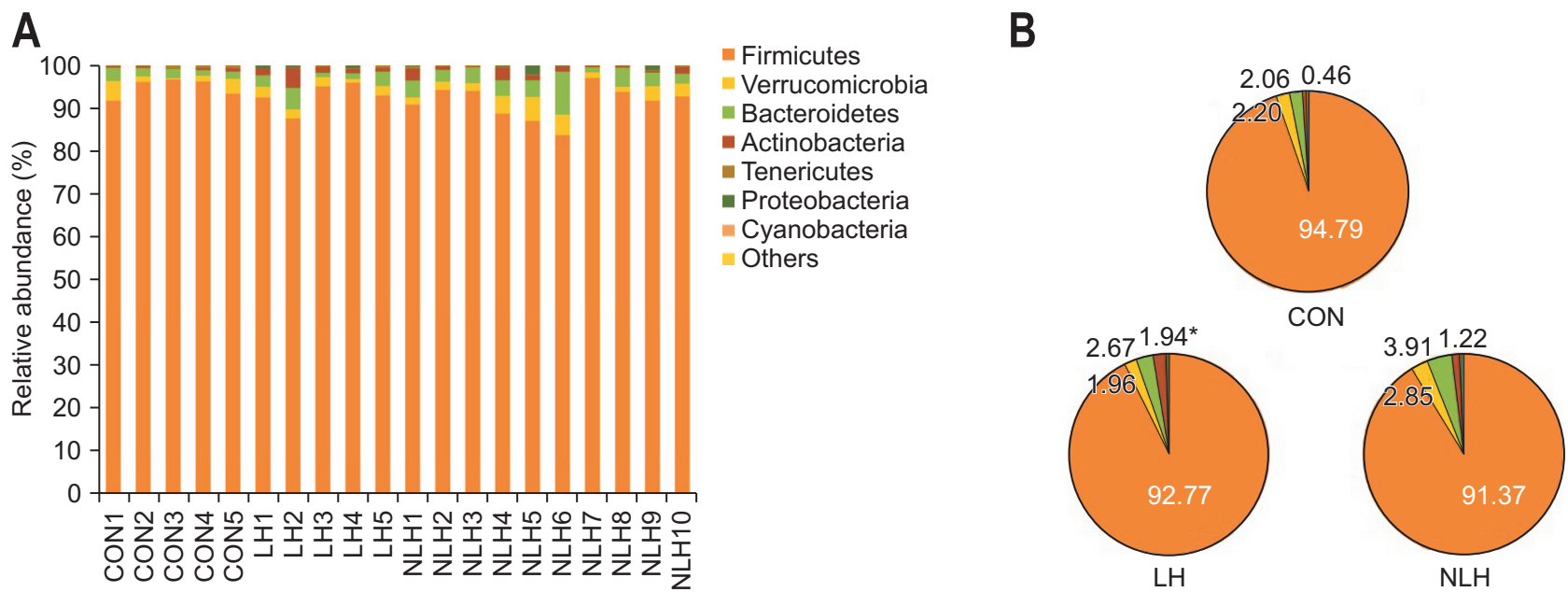

Fig. 2. Comparison of gut microbiota composition at the phylum level in rats from each group. (A) Variation in gut microbiota composition in the cecum at the phylum level in each rat (CON: 5 control rats subjected to neither foot shock nor the avoidance/escape task procedure; LH: 5 rats diagnosed with LH; NLH: 10 rats diagnosed as not having LH). (B) Relative abundance of gut microbiota composition in the cecum at the phylum level in rats from each group. The relative abundance of Actinobacteria was significantly increased in the LH group compared to that in the control group: $1.94 \% \pm 0.72 \%$ in the LH group, $1.22 \% \pm 0.32 \%$ in the NLH group, and $0.46 \% \pm 0.11 \%$ in the control group. The relative abundance of Firmicutes was $92.78 \% \pm 1.46 \%$ in the LH group, $91.37 \% \pm 1.24 \%$ in the NLH group, and $94.79 \% \pm 0.98 \%$ in the control group. The relative abundance of Verrucomicrobia was 1.96\% $0.30 \%$ in the LH group, $2.85 \% \pm 0.49 \%$ in the NLH group, and $2.20 \% \pm 0.80 \%$ in the control group. The relative abundance of Bacteroidetes was 2.67\% $0.72 \%$ in the LH group, 3.91\% $\% 0.75 \%$ in the NLH group, and 2.06\% $0.31 \%$ in the control group. ${ }^{*} \mathrm{p}<0.05$ vs control rats. p-values obtained via the Kruskal-Wallis test followed by the Steel-Dwass post hoc test. 


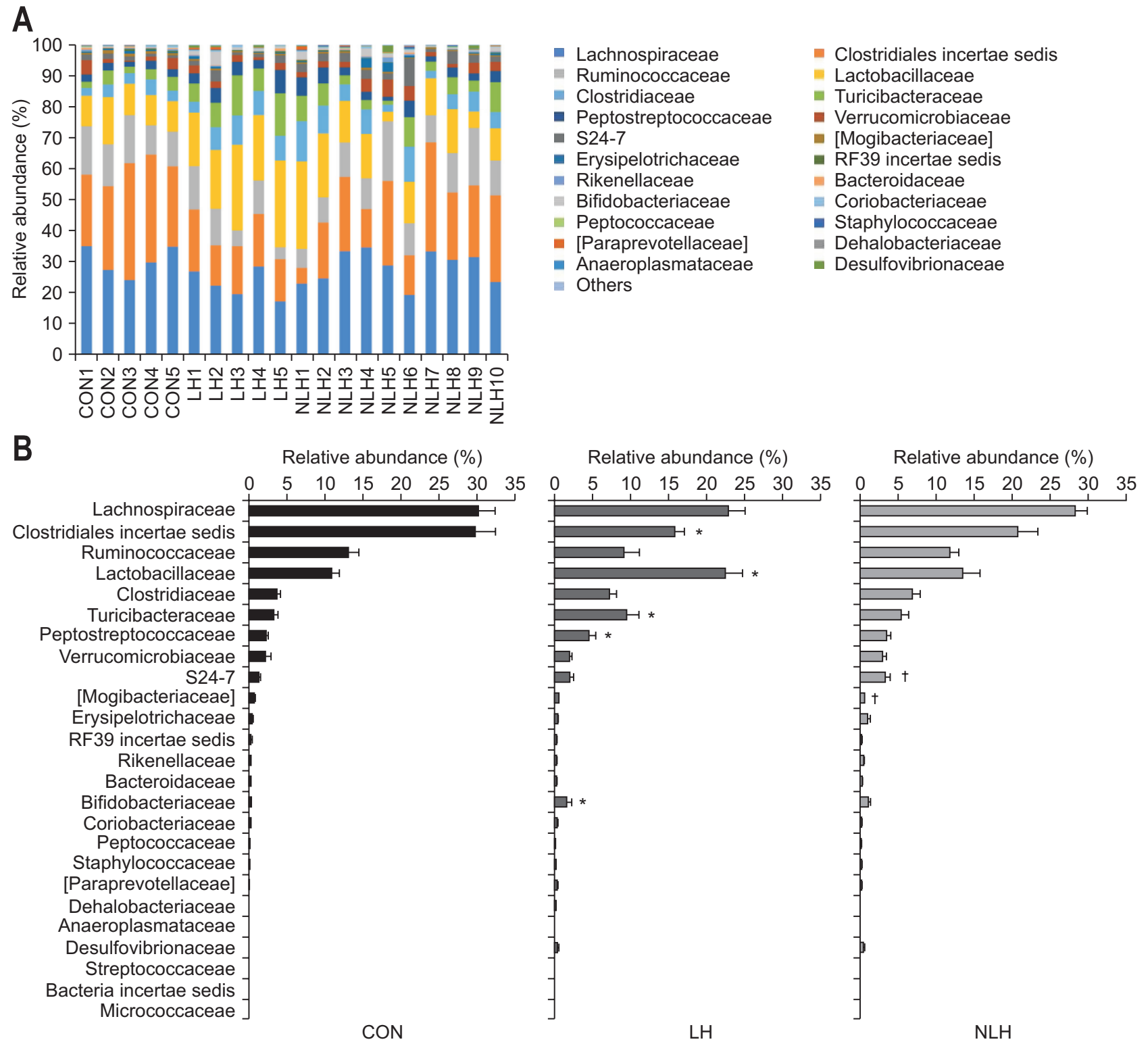

Fig. 3. Comparison of gut microbiota composition at the family level in rats from each group. (A) Variation in gut microbiota composition in the cecum at the family level in each rat (CON: 5 control rats subjected to neither foot shock nor the avoidance/escape task procedure; LH: 5 rats diagnosed with learned helplessness (LH); NLH: 10 rats diagnosed as not having LH). (B) Relative abundance of gut microbiota composition in the cecum at the family level in rats from each group. ${ }^{*} \mathrm{p}<0.05$ vs control rats. $p$-values obtained via the Kruskal-Wallis test followed by the SteelDwass post hoc test. ${ }^{\dagger} \mathrm{p}<0.05$ vs. control rats.

trol group (Table 1). The relative abundance of S24-7 incertae sedis was significantly higher in the non-LH group than in the control group, while that of Mogibacteriaceae incertae sedis was significantly lower in the non-LH group than in the control group (Table 1).

\section{The diversity of the gut microbiota is altered in rats with} LH

Next, we compared the $\alpha$-diversity of the cecal microbiota in each group by determining Shannon index values (Fig. 4). The value in the LH group tended to be lower than those in the other groups. However, there were no significant differences in the values among the three groups (Fig. 4). Furthermore, we analyzed the $\beta$-diversity of the cecal microbiota in each group by performing principle coordinate analysis of the weighted UniFrac distances of the 16S rRNA genes (Fig. 5). The analysis showed that the distribution pattern of the values was clearly different between the LH and control groups (Fig. 5).

\section{Neither visceral hypersensitivity nor the composition of the gut microbiota is altered in rats with PTSD}

Thorough analysis of the behavior of the rats in the non-LH group showed that some of the rats could be diagnosed with PTSD. Therefore, we classified the non-LH group rats into a 
Table 1. Mean Relative Abundance of Major Cecal Microbiota (>0.1\%) at the Genus Level

\begin{tabular}{|c|c|c|c|c|c|c|}
\hline Genus & CON (\%) & LH (\%) & NLH (\%) & $\begin{array}{c}\text { Kruskal-Wallis } \\
\text { p-value }\end{array}$ & \multicolumn{2}{|c|}{$\begin{array}{c}\text { Steel-Dwass } \\
\text { p-value }\end{array}$} \\
\hline Clostridiales incertae sedis & $29.6665 \pm 2.79$ & $15.8018 \pm 1.24$ & $20.7234 \pm 2.80$ & 0.0407 & CON vs LH & 0.0326 \\
\hline Lachnospiraceae incertae sedis & $17.0738 \pm 2.68$ & $10.0771 \pm 1.15$ & $13.7927 \pm 1.32$ & 0.1014 & & \\
\hline Lactobacillus & $10.8927 \pm 1.07$ & $22.5036 \pm 2.20$ & $13.4452 \pm 2.25$ & 0.0167 & CON vs LH & 0.0326 \\
\hline Oscillospira & $7.7237 \pm 1.20$ & $3.2864 \pm 1.14$ & $6.3002 \pm 1.02$ & 0.0500 & & \\
\hline [Ruminococcus] & $5.5351 \pm 0.77$ & $5.0135 \pm 0.58$ & $5.9964 \pm 0.48$ & 0.7007 & & \\
\hline Coprococcus & $5.1522 \pm 1.76$ & $5.9013 \pm 1.53$ & $6.0364 \pm 0.65$ & 0.5761 & & \\
\hline Turicibacter & $3.2801 \pm 0.54$ & $9.5465 \pm 1.59$ & $5.3737 \pm 0.96$ & 0.0292 & CON vs LH & 0.0326 \\
\hline Ruminococcus & $3.1728 \pm 0.21$ & $4.2252 \pm 1.26$ & $3.3148 \pm 0.32$ & 0.8258 & & \\
\hline Peptostreptococcaceae incertae sedis & $2.3304 \pm 0.20$ & $4.5191 \pm 0.83$ & $3.3783 \pm 0.50$ & 0.0485 & CON vs LH & 0.0326 \\
\hline Clostridium & $2.1533 \pm 0.31$ & $3.8920 \pm 0.74$ & $4.1808 \pm 0.96$ & 0.4089 & & \\
\hline Ruminococcaceae incertae sedis & $1.7582 \pm 0.23$ & $1.3899 \pm 0.31$ & $1.6280 \pm 0.18$ & 0.4909 & & \\
\hline Roseburia & $1.6500 \pm 0.23$ & $0.9156 \pm 0.33$ & $1.4071 \pm 0.31$ & 0.3253 & & \\
\hline SMB53 & $1.4993 \pm 0.25$ & $3.2816 \pm 0.81$ & $2.5161 \pm 0.37$ & 0.1365 & & \\
\hline S24-7 incertae sedis & $1.3917 \pm 0.20$ & $1.9529 \pm 0.49$ & $3.1707 \pm 0.69$ & 0.0309 & $\mathrm{CON}$ vs NLH & 0.0446 \\
\hline [Mogibacteriaceae] incertae sedis & $0.7687 \pm 0.10$ & $0.4628 \pm 0.03$ & $0.4052 \pm 0.04$ & 0.0151 & CON vs NLH & 0.0230 \\
\hline Blautia & $0.5414 \pm 0.26$ & $0.6664 \pm 0.42$ & $0.7713 \pm 0.36$ & 0.8907 & & \\
\hline RF39 incertae sedis & $0.3690 \pm 0.10$ & $0.2143 \pm 0.09$ & $0.1640 \pm 0.04$ & 0.2683 & & \\
\hline Rikenellaceae incertae sedis & $0.3055 \pm 0.10$ & $0.1836 \pm 0.07$ & $0.3499 \pm 0.14$ & 0.6917 & & \\
\hline Butyricicoccus & $0.2925 \pm 0.16$ & $0.1359 \pm 0.07$ & $0.2645 \pm 0.06$ & 0.6878 & & \\
\hline Bacteroides & $0.2730 \pm 0.07$ & $0.2047 \pm 0.12$ & $0.1410 \pm 0.04$ & 0.1566 & & \\
\hline Erysipelotrichaceae incertae sedis & $0.2204 \pm 0.09$ & $0.2157 \pm 0.14$ & $0.1205 \pm 0.03$ & 0.7579 & & \\
\hline Adlercreutzia & $0.1956 \pm 0.09$ & $0.3427 \pm 0.08$ & $0.2090 \pm 0.05$ & 0.2505 & & \\
\hline Clostridium & $0.1533 \pm 0.07$ & $0.0379 \pm 0.02$ & $0.0516 \pm 0.02$ & 0.4385 & & \\
\hline Clostridium & $0.1147 \pm 0.03$ & $0.0524 \pm 0.02$ & $0.0835 \pm 0.02$ & 0.2505 & & \\
\hline Clostridium & $0.1021 \pm 0.03$ & $0.0739 \pm 0.04$ & $0.0691 \pm 0.01$ & 0.6542 & & \\
\hline [Eubacterium] & $0.1010 \pm 0.07$ & $0.0435 \pm 0.04$ & $0.0178 \pm 0.01$ & 0.2421 & & \\
\hline
\end{tabular}

Data are presented as mean \pm SEM. CON $(n=5), \mathrm{LH}(\mathrm{n}=5)$, and NLH $(\mathrm{n}=10)$.

CON, control group; LH, learned helplessness group; NLH, non-LH group.

Kruskal-Wallis test followed by the Steel-Dwass post hoc test.

PTSD group and a non-PTSD group and compared these groups using the same approach as for the LH comparisons. We observed no significant differences in threshold CRD values among the control, PTSD, and non-PTSD groups (Supplementary Fig. 1). There were also no significant differences in relative abundances of microbes at the phylum level among the three groups (Supplementary Fig. 2). High-throughput microbial community analysis of the cecal microbiota at the family level in each rat showed that the relative abundance of S24-7 was significantly higher in the PTSD group than in the control group (Supplementary Fig. 3). In addition, the relative abundance of Mogibacteriaceae was significantly lower in the non-PTSD group than in the control group (Supplementary Fig. 3).

Furthermore, we identified the rat cecal microbes with relative abundances of $0.1 \%$ or greater at the genus level (Supplemen- tary Table 1). The relative abundance of S24-7 incertae sedis was significantly higher in the PTSD group than in the control group (Supplementary Table 1), and the relative abundance of Mogibacteriaceae incertae sedis was significantly lower in the non-PTSD group than in the control and PTSD groups (Supplementary Table 1).

\section{DISCUSSION}

In the present study, we found that threshold CRD values were significantly lower in LH group rats, which are thought to model depression, than in control group rats. Visceral hypersensitivity, which corresponds to low CRD values, is known to be a hallmark of IBS. ${ }^{18,19}$ Our results support those of previous reports showing that individuals with a history of depression 
are at high risk of developing IBS. ${ }^{1,2}$ In our study, after undergoing the same stress, rats that did and did not develop depression were examined and compared. We found that IBS significantly

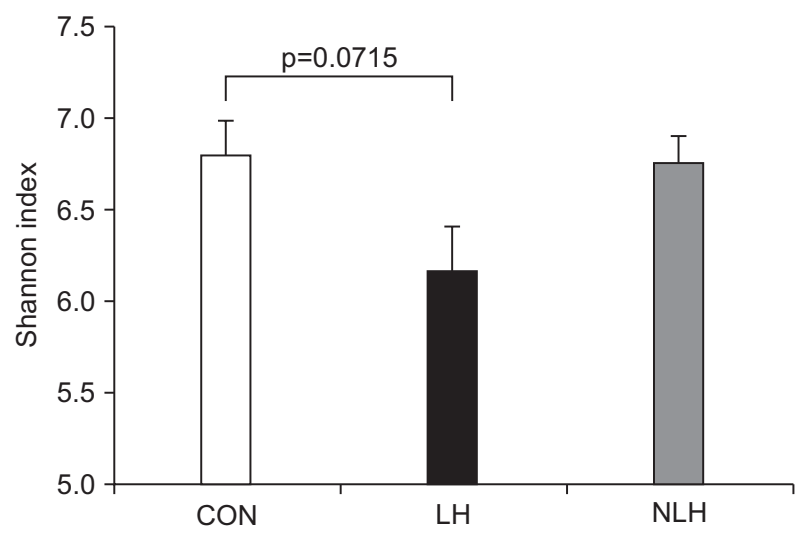

Fig. 4. The $\alpha$-diversity of the gut microbiota in rats from each group. $\alpha$-Diversity of the cecal microbiota in each group (CON: 5 control rats subjected to neither foot shock nor the avoidance/escape task procedure; LH: 5 rats diagnosed with learned helplessness (LH); NLH: 10 rats diagnosed as not having $\mathrm{LH}$ ), as indicated by the values of the Shannon index. p-values obtained via one-way analysis of variance with Tukey post hoc test. co-occurred in rats that developed depression. The relationship between depression and gut function has previously been evaluated in some animal models. For example, Zhang et al. ${ }^{20}$ reported greater contractile force of colonic strips in a depressive rat model induced by chronic unpredictable mild stress than in control rats. Furthermore, Park et al. ${ }^{21}$ showed that colonic motor activity and the composition of the gut microbiota were altered in a mouse model of depression induced by bilateral olfactory bulbectomy. Our findings provide new animal modelbased evidence that depression itself, rather than specific stressors, causes the onset of IBS.

In recent years, it has been reported that changes in intestinal flora are involved in the onset of IBS. ${ }^{22}$ It has also been reported that gut microbiome alterations can be important etiological factors underlying depression. ${ }^{6}$ Therefore, we performed highthroughput microbial community analysis of the cecal microbiota in our rat model. The present study showed that the relative abundance of Clostridiales incertae sedis, the most prevalent microbe group, was significantly lower in depressed rats than in control rats. Recent reports have shown that the relative abundance of Clostridiales incertae sedis in the gut microbiota is significantly lower in chronic variable stress-induced rat models of
A

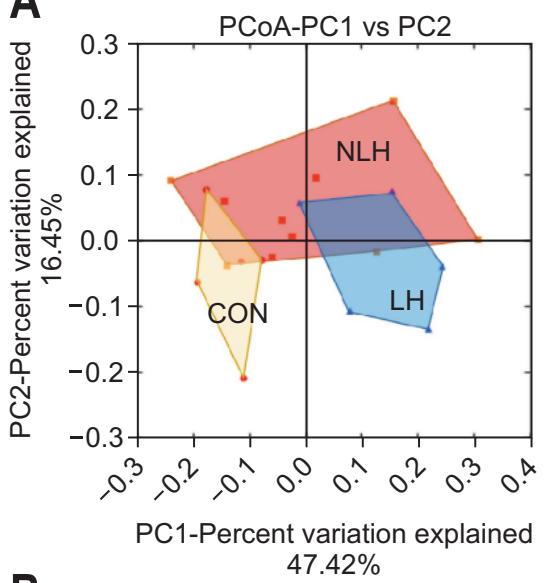

B

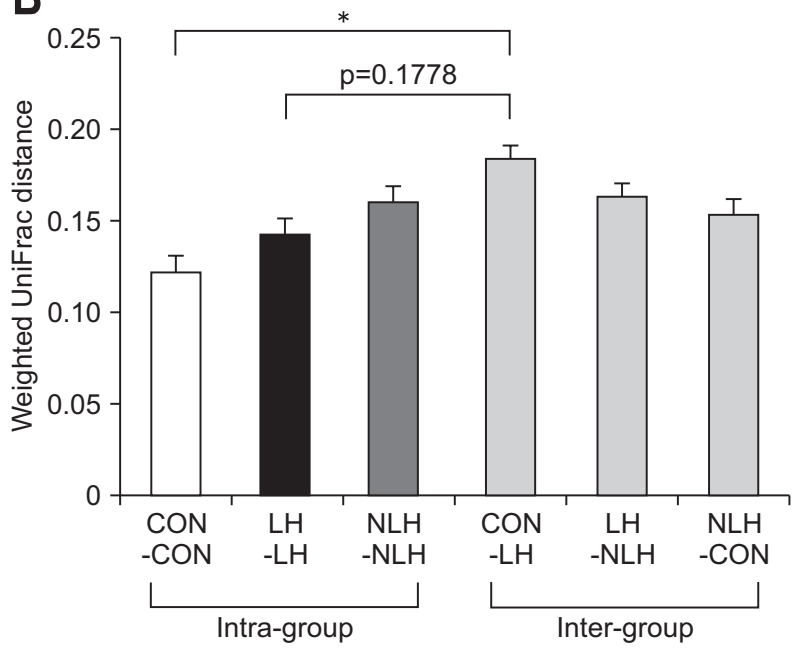

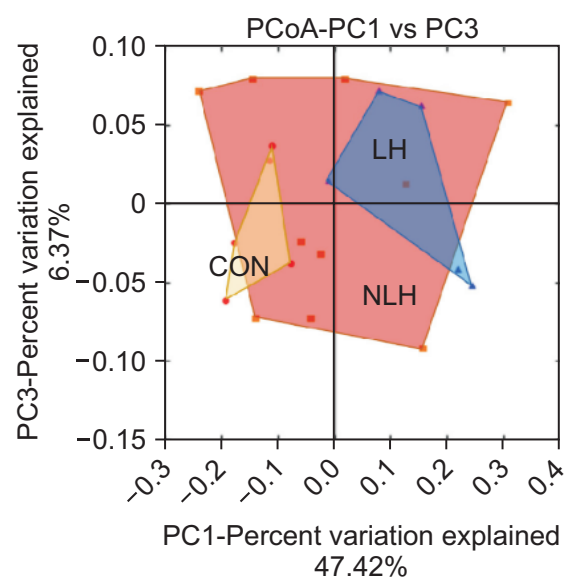

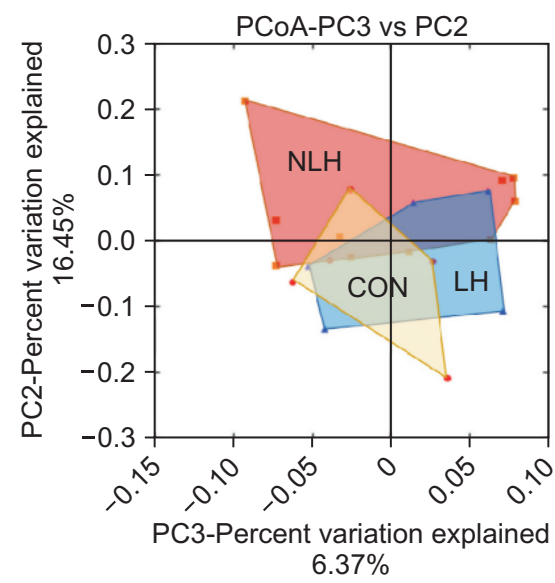

Fig. 5. The $\beta$-diversity of the gut microbiota in rats from each group. (A) $\beta$-Diversity of the cecal microbiota in each group (CON: 5 control rats subjected to neither foot shock nor the avoidance/escape task procedure; LH: 5 rats diagnosed with learned helplessness (LH); NLH: 10 rats diagnosed as not having $\mathrm{LH}$ ), represented by a 2-dimensional graph obtained using principal coordinate analysis (PCoA) of weighted UniFrac distances of $16 \mathrm{~S}$ rRNA genes. (B) Weighted UniFrac distances within and between groups. ${ }^{*} \mathrm{p}<0.01$ vs control intragroup. p-values obtained via one-way analysis of variance with Tukey post hoc test. 
depression than in control rats. ${ }^{23}$ Furthermore, a recent human study showed that IBS symptom severity is negatively associated with enterotypes enriched with Clostridiales species. ${ }^{4}$ These results suggest that depression-induced decreases in Clostridiales species may cause IBS.

The present study also showed that the relative abundances of Lactobacillus, Turicibacter, Peptostreptococcaceae incertae sedis, and Bifidobacterium were significantly higher in depressed rats than in control rats. These results do not seem to be compatible with those of a recent study showing that the colonization of Lactobacillus and Bifidobacterium bacteria is significantly lower in depressed patients than in control subjects. ${ }^{24,25}$ PintoSanchez et al. ${ }^{26}$ also reported that a probiotic, Bifidobacterium longum NCC3001, ameliorates comorbid depression and affects the activity of multiple brain areas involved in emotion. On the other hand, a recent study has shown that Lactobacillus and Turicibacter are more abundant in Wistar-Kyoto rats, which are known to exhibit depression-like phenotypes correlated with stress-associated functional gastrointestinal disorders, than they are in control Sprague-Dawley rats. ${ }^{27}$ Labus et al. ${ }^{28}$ also reported that patients with IBS have a greater relative abundance of Lactobacillales and that Lactobacillales are positively correlated with a history of early life trauma. Furthermore, they showed a positive association between gut microbiota composition and regional brain structural changes in IBS. These previous findings, taken together with the results of the present study, suggest that intestinal dysbiosis along with decreases in Clostridiales species and increases in Lactobacillus and Bifidobacterium species may be characteristics of concurrent depression and IBS.

Furthermore, our results showed that the diversity of the gut microbiota was altered in depressed rats, supporting recent reports that depression and IBS are associated with decreased gut microbiota diversity. ${ }^{29,30}$

Finally, the present study showed that rats with PTSD, unlike depressed rats, did not have increased visceral hypersensitivity or significant changes in the gut microbiome. This suggests that visceral sensitivity and intestinal bacterial flora could differ among psychiatric disorders.

There are some limitations to this study. First, we did not evaluate the expression of corticotrophin-releasing hormone, which is known to be related to the hypothalamic-pituitaryadrenocortical axis in IBS. Second, we did not examine colonic 5-hydroxytryptamine levels or c-Fos expression, which could play a role in the pathogenesis of IBS. Such examination would be helpful to clarify the role of the brain-gut axis in our rat model of depression coinciding with IBS.

In conclusion, our rat model of depression is useful for clarifying the effect of depression on the onset of IBS. Our findings imply that the development of depression itself, rather than specific stressors, promotes the onset of IBS. The present study also shows that individual psychiatric diseases, viz., depression and PTSD, have unique intestinal flora profiles and that dysbiosis, such as decreases in Clostridiales species, accompanying the onset of depression could promote the development of IBS. In the future, it is expected that the brain-gut interaction mechanism will be further elucidated through examination of the intestina flora of patients with coincident depression and IBS. Such studies may eventually lead to the development of novel therapeutic strategies.

\section{CONFLICTS OF INTEREST}

No potential conflict of interest relevant to this article was reported.

\section{ACKNOWLEDGEMENTS}

This work was supported in part by a grant from National Defense Medical College.

Author contributions: T. Takajo, K. Tomita, H. Tsuchihashi, K. Shimizu, and R. Hokari designed the experiments. T. Takajo, H. Tsuchihashi, S. Enomoto, M. Tanichi, H. Toda, Y. Okada, H. Furuhashi, N. Sugihara, A. Wada, and K. Shimizu performed the experiments. T. Takajo, K. Tomita, H. Tsuchihashi, K. Horiuchi, K. Inaba, Y. Hanawa, N. Shibuya, K. Shirakabe, M. Higashiyama, C. Kurihara, C. Watanabe, S. Komoto, S. Nagao, K. Kimura, S. Miura, K. Shimizu, and R. Hokari were involved in the interpretation of data. T. Takajo, K. Tomita, and K. Shimizu drafted the manuscript.

\section{REFERENCES}

1. Kanazawa M, Endo Y, Whitehead WE, Kano M, Hongo M, Fukudo S. Patients and nonconsulters with irritable bowel syndrome reporting a parental history of bowel problems have more impaired psychological distress. Dig Dis Sci 2004;49:1046-1053.

2. Koloski NA, Jones M, Kalantar J, Weltman M, Zaguirre J, Talley NJ. The brain: gut pathway in functional gastrointestinal disorders is bidirectional. A 12-year prospective population-based study. Gut 2012;61:1284-1290.

3. Grenham S, Clarke G, Cryan JF, Dinan TG. Brain-gut-microbe communication in health and disease. Front Physiol 2011;2:94.

4. Tap J, Derrien M, Törnblom H, et al. Identification of an intestinal microbiota signature associated with severity of irritable bowel syndrome. Gastroenterology 2017;152:111-123.

5. Rodiño-Janeiro BK, Vicario M, Alonso-Cotoner C, Pascua-García $\mathrm{R}$, Santos J. A review of microbiota and irritable bowel syndrome: future in therapies. Adv Ther 2018;35:289-310.

6. Lima-Ojeda JM, Rupprecht R, Baghai TC. "I am I and my bacterial circumstances": linking gut microbiome, neurodevelopment, and depression. Front Psychiatry 2017;8:153.

7. Koba T, Kodama Y, Shimizu K, et al. Persistent behavioural changes in rats following inescapable shock stress: a potential model of posttraumatic stress disorder. World J Biol Psychiatry 2001;2:34- 
37.

8. Sawamura T, Shimizu K, Nibuya M, et al. Effect of paroxetine on a model of posttraumatic stress disorder in rats. Neurosci Lett 2004;357:37-40.

9. Wakizono T, Sawamura T, Shimizu K, et al. Stress vulnerabilities in an animal model of post-traumatic stress disorder. Physiol Behav 2007;90:687-695.

10. Takahashi T, Shimizu K, Shimazaki K, Toda H, Nibuya M. Environmental enrichment enhances autophagy signaling in the rat hippocampus. Brain Res 2014;1592:113-123.

11. Kikuchi A, Shimizu K, Nibuya M, et al. Relationship between posttraumatic stress disorder-like behavior and reduction of hippocampal 5-bromo-2'-deoxyuridine-positive cells after inescapable shock in rats. Psychiatry Clin Neurosci 2008;62:713-720.

12. Overmier JB, Seligman ME. Effects of inescapable shock upon subsequent escape and avoidance responding. J Comp Physiol Psychol 1967;63:28-33.

13. Musty RE, Jordan MP, Lenox RH. Criterion for learned helplessness in the rat: a redefinition. Pharmacol Biochem Behav 1990;36:739-744.

14. Al-Chaer ED, Kawasaki M, Pasricha PJ. A new model of chronic visceral hypersensitivity in adult rats induced by colon irritation during postnatal development. Gastroenterology 2000;119:12761285.

15. Jinno S, Toshimitsu T, Nakamura Y, et al. Maternal prebiotic ingestion increased the number of fecal bifidobacteria in pregnant women but not in their neonates aged one month. Nutrients 2017;9:E196.

16. Caporaso JG, Lauber CL, Walters WA, et al. Ultra-high-throughput microbial community analysis on the Illumina HiSeq and MiSeq platforms. ISME J 2012;6:1621-1624.

17. Caporaso JG, Kuczynski J, Stombaugh J, et al. QIIME allows analysis of high-throughput community sequencing data. Nat Methods 2010;7:335-336.

18. Keszthelyi D, Troost FJ, Masclee AA. Irritable bowel syndrome: methods, mechanisms, and pathophysiology. Methods to assess visceral hypersensitivity in irritable bowel syndrome. Am J Physiol Gastrointest Liver Physiol 2012;303:G141-G154.

19. Ludidi S, Mujagic Z, Jonkers D, et al. Markers for visceral hyper- sensitivity in patients with irritable bowel syndrome. Neurogastroenterol Motil 2014;26:1104-1111.

20. Zhang L, Hu L, Chen M, Yu B. Exogenous interleukin-6 facilitated the contraction of the colon in a depression rat model. Dig Dis Sci 2013;58:2187-2196.

21. Park AJ, Collins J, Blennerhassett PA, et al. Altered colonic function and microbiota profile in a mouse model of chronic depression. Neurogastroenterol Motil 2013;25:733-e575.

22. Gwee KA, Ghoshal UC, Chen M. Irritable bowel syndrome in Asia: pathogenesis, natural history, epidemiology, and management. J Gastroenterol Hepatol 2018;33:99-110.

23. Yu M, Jia H, Zhou C, et al. Variations in gut microbiota and fecal metabolic phenotype associated with depression by $16 \mathrm{~S}$ rRNA gene sequencing and LC/MS-based metabolomics. J Pharm Biomed Anal 2017;138:231-239.

24. Aizawa E, Tsuji H, Asahara T, et al. Possible association of Bifidobacterium and Lactobacillus in the gut microbiota of patients with major depressive disorder. J Affect Disord 2016;202:254-257.

25. Fung TC, Olson CA, Hsiao EY. Interactions between the microbiota, immune and nervous systems in health and disease. Nat Neurosci 2017;20:145-155.

26. Pinto-Sanchez MI, Hall GB, Ghajar K, et al. Probiotic Bifidobacterium longum NCC3001 reduces depression scores and alters brain activity: a pilot study in patients with irritable bowel syndrome. Gastroenterology 2017;153:448-459.

27. Dalziel JE, Fraser K, Young W, McKenzie CM, Bassett SA, Roy NC. Gastroparesis and lipid metabolism-associated dysbiosis in WistarKyoto rats. Am J Physiol Gastrointest Liver Physiol 2017;313:G62G72.

28. Labus JS, Hollister EB, Jacobs J, et al. Differences in gut microbial composition correlate with regional brain volumes in irritable bowel syndrome. Microbiome 2017;5:49.

29. Kelly JR, Borre Y, O’ Brien C, et al. Transferring the blues: depression-associated gut microbiota induces neurobehavioural changes in the rat. J Psychiatr Res 2016;82:109-118.

30. Mosca A, Leclerc M, Hugot JP. Gut microbiota diversity and human diseases: should we reintroduce key predators in our ecosystem? Front Microbiol 2016;7:455. 